\title{
IUCN
}

\section{IUCN Review Protocol for Biodiversity Net Gain}

A guide for undertaking independent reviews of progress towards a net gain for biodiversity

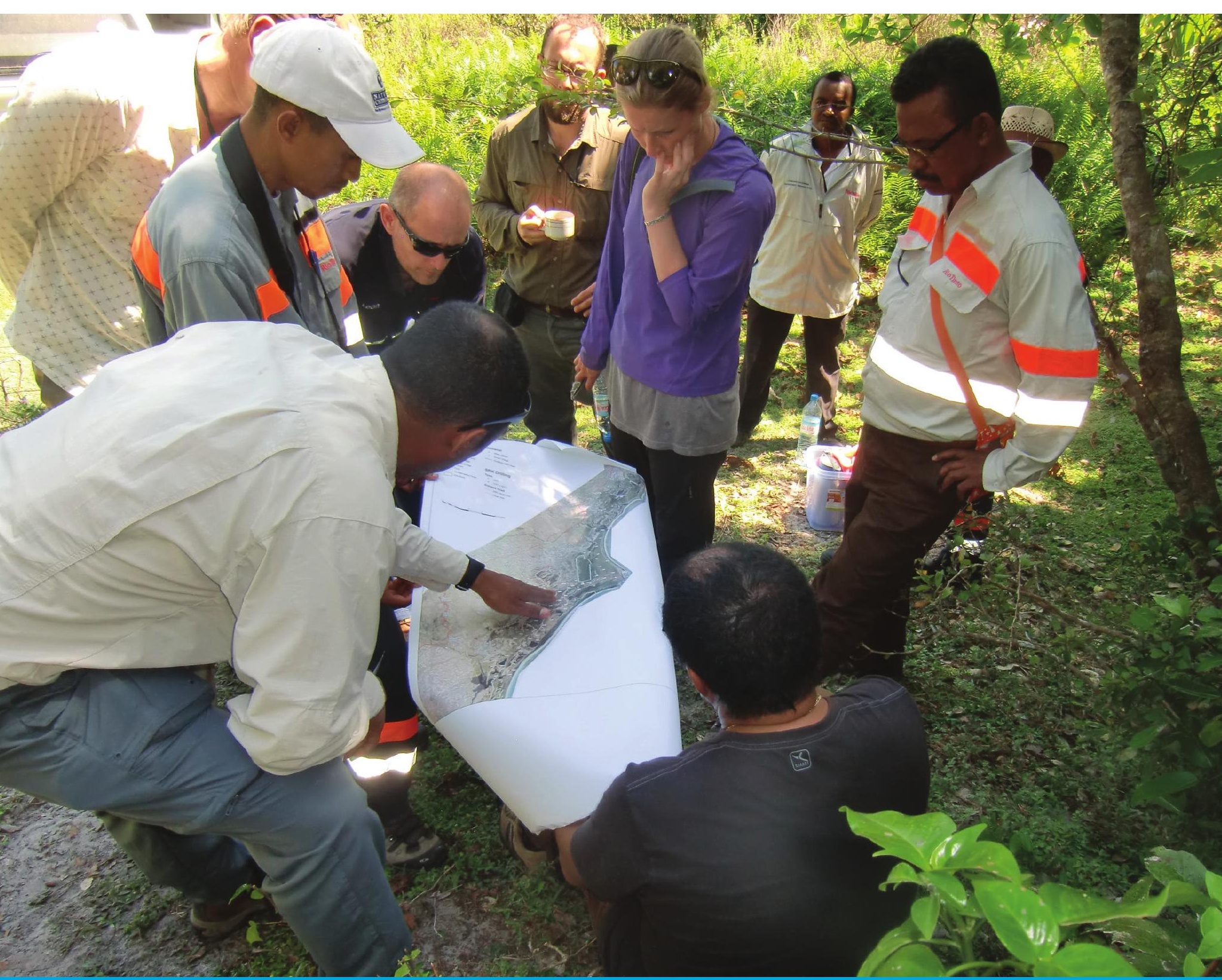




\section{About IUCN}

IUCN is a membership Union uniquely composed of both government and civil society organisations. It provides public, private and non-governmental organisations with the knowledge and tools that enable human progress, economic development and nature conservation to take place together.

Created in 1948, IUCN is now the world's largest and most diverse environmental network, harnessing the knowledge, resources and reach of 1,300 member organisations and some 16,000 experts. It is a leading provider of conservation data, assessments and analysis. Its broad membership enables IUCN to fill the role of incubator and trusted repository of best practices, tools and international standards.

IUCN provides a neutral space in which diverse stakeholders including governments, NGOs, scientists, businesses, local communities, indigenous peoples organisations and others can work together to forge and implement solutions to environmental challenges and achieve sustainable development.

Working with many partners and supporters, IUCN implements a large and diverse portfolio of conservation projects worldwide. Combining the latest science with the traditional knowledge of local communities, these projects work to reverse habitat loss, restore ecosystems and improve people's well-being.

www.iucn.org 


\section{IUCN Review Protocol for Biodiversity Net Gain}

A guide for undertaking independent reviews of progress towards a net gain for biodiversity 
The designation of geographical entities in this book, and the presentation of the material, do not imply the expression of any opinion whatsoever on the part of IUCN concerning the legal status of any country, territory, or area, or of its authorities, or concerning the delimitation of its frontiers or boundaries.

Published by IUCN, Gland, Switzerland

Copyright (C) 2017 International Union for Conservation of Nature and Natural Resources

Reproduction of this publication for educational or other non-commercial purposes is authorized without prior written permission from the copyright holder provided the source is fully acknowledged.

Reproduction of this publication for resale or other commercial purposes is prohibited without prior written permission of the copyright holder.

Citation IUCN Business and Biodiversity Programme (2017). IUCN Review Protocol for Biodiversity Net Gain: A guide for undertaking independent reviews of progress towards a net gain for biodiversity. Gland, Switzerland: IUCN. 32pp.

DOI 10.2305/IUCN.CH.2017.08.en

Cover photo Stephen Edwards

Layout by Stéfane Mauris, ROSAFILMS

Available from IUCN (International Union for Conservation of Nature)

Business and Biodiversity Programme

Rue Mauverney 28

1196 Gland, Switzerland

Tel +41229990000

Fax +41229990002

www.iucn.org/resources/publications 


\section{Contents}

ACKNOWLEDGEMENTS $\quad 6$

$\begin{array}{ll}\text { EXECUTIVE SUMMARY } & 7\end{array}$

1. OVERVIEW OF THE IUCN BIODIVERSITY NET GAIN REVIEW PROTOCOL 8

2. THE BNG REVIEW PROTOCOL 10

2.1.What is BNG? 10

2.2.What is the BNG Review Protocol?

2.3.How should this BNG Review Protocol be used? 12

3. PREPARING FOR A REVIEW 13

3.1.Before the review 13

3.2. The Team 13

3.3. The Review Process 15

4. VISIT 18

5. POST-VISIT 20

6. GLOSSARY 22

7. ANNEXES 26

7.1. Terms of Reference for a BNG Review Team 26

7.2. Terms of Reference for a BNG Review Team Lead 28

7.3.Terms of Reference for a BNG Review Team Member 30 


\section{Acknowledgements}

IUCN Review Protocol for Biodiversity Net Gain would not have been possible without the experience gained from working with Rio Tinto, which piloted the application of a Net Positive Impact (NPI) approach at several sites from 2011-2014.

This work also builds on a two-year collaborative effort on NPI, under the auspices of the NPI Alliance, which included IUCN, Rio Tinto, Shell and The Nature Conservancy. IUCN is extremely grateful to individuals within these organisations, who generated new knowledge around Biodiversity Net Gain (BNG) for business and conservation, and ultimately, helped inform IUCN's strategy moving forward.

More specifically, the authors and contributors to the original NPI Panel and draft protocols, which lead to this publication, are listed in alphabetic order with their affiliation at time of their involvement:

\author{
Stuart Anstee (Rio Tinto) \\ Rachel Asante-Owusu (IUCN) \\ Monica Barcellos Harris (IUCN/UNEP-WCMC) \\ Leon Bennun (formerly BirdLife International) \\ Gerard Bos (IUCN) \\ Thomas Brooks (NatureServe) \\ Stephen Edwards (IUCN) \\ Dennis Hosack (IUCN/Rio Tinto) \\ Pippa Howard (Fauna \& Flora International) \\ Sam Luccitti (Rio Tinto)
}

\author{
Sally Madden (Rio Tinto) \\ Leon Payne (Rio Tinto) \\ Conrad Savy (formerly an independent \\ consultant) \\ Rainer Schneeweiss (Rio Tinto) \\ Melissa Tolley (UNEP-WCMC) \\ Manon Vincelette (Rio Tinto) \\ James Watson (Wildlife Conservation Society/ \\ University of Queensland) \\ Samir Whitaker (BirdLife International).
}

Finally, this protocol is a reflection of a much larger body of work focused on engaging business in biodiversity conservation, and therefore, IUCN would like to acknowledge these contributions as well. 


\section{Executive Summary}

From engaging on biodiversity conservation projects and developing new science-based tools, IUCN is committed to working with business to advance our common objectives on nature, knowledge sharing and good practice.

This latest guide, the IUCN Review Protocol for Biodiversity Net Gain, aims to assist companies, which are interested in transforming their operations to meet today's widely-accepted global goals for biodiversity and sustainable development.

In many forward-looking companies, projects are already required to apply systematic procedures to avoid, minimise, rehabilitate and offset, if possible, any residual impacts to biodiversity on site. This decision-making process, known as the mitigation hierarchy, is increasingly being adopted by companies and governments as a standard that not only enhances environmental management, but also contributes to ensuring a biodiversity net gain.

As IUCN describes it, the difference between no net loss and biodiversity net gain are determined by the level of ambition in applying the mitigation hierarchy. In some cases, the development project goal is to achieve a no net loss of biodiversity and it others, it is to ensure biodiversity gain significantly exceed the loss, in which case the term 'net gain' may be used.

The IUCN Review Protocol provides a step-bystep guide for project managers to measure their progress on achieving their biodiversity net gain targets. The Protocol describes a robust process for preparing for such a review, as well as the components required to ensure such an assessment is rigorous yet responsive to ongoing developments throughout the project lifespan.
After working with partners in the extractives industry to apply a net positive impact approach, as well as piloting this approach with the agriculture and forestry sectors, IUCN is convinced that companies can achieve a no net loss in biodiversity at the very least, and in most cases, a biodiversity net gain.

This Protocol reflects input from numerous IUCN colleagues, partners and experts, who are actively engaged in on-site biodiversity management. In particular, IUCN's partnership with Rio Tinto, where a similar methodology was tested on three mine sites, has been invaluable to informing this process. The knowledge gained from this practical experience, as well as from other corporate partners, the International Finance Corporation, IUCN Members and Commissions, and others who participated in consultations at the 2016 IUCN World Conservation Congress, have all played a key role in shaping this guide.

The IUCN Review Protocol for Biodiversity Net Gain aims to contribute to a growing understanding and series of tools that can assist business and governments committed to safeguarding nature. While such reviews are not yet commonplace, IUCN envisions that one day soon ensuring a net gain for biodiversity will be standard practice for all. 


\title{
1. Overview of the IUCN Biodiversity Net Gain Review Protocol
}

\section{Objective}

\author{
The IUCN Biodiversity Net Gain (BNG) Review Protocol is an \\ independent evaluation tool for assessing the planning and \\ progress towards achieving a net gain for biodiversity, as well as \\ serves as a guide for identifying any potential limits for \\ implementing BNG at a particular project.
}

The IUCN Review Protocol is a robust and tested process that project reviewers and assessors can use to evaluate projects that have adopted a biodiversity net gain target. While undertaking a BNG Review includes several components (as illustrated in the below box), it is strongly recommended that prior to the first application of the Review Protocol, to a project spend time on the customization and development of a project-specific, fit-for-purpose approach. No two projects are exactly alike, but as more companies and governments strive to achieve BNG, the Protocol is a tool that can help measure their efforts and ultimate success.

\section{Minimum requirements for a BNG Review include:}

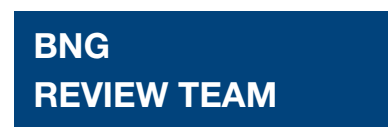

- Expertise in natural and/or social science aspects of biodiversity conservation.

- Disclosed conflict of interest.

- At least one member with siterelevant biodiversity conservation experience.

- Balanced by gender and other locally appropriate considerations.

- BNG Team Lead: prior experience with leading BNG Reviews and leadership of technical teams and/or evaluation processes.

MINIMUM
INFORMATION
- An assessment
of project risks
and impacts on
biodiversity.
- A forecast (qualitative
or quantitative) of the
process and timing of
BNG achievement.
- A management plan
that describes detailed
objectives, tasks,
timelines, and tasks
owners related to
BNG-related activities.
- A Biodiversity Offset
Plan, in cases where
offsets are considered.

\section{PROJECT \\ REPRESENTATIVE}

- Liaison between BNG Team and project, including logistics management.

- Knowledge of BNGrelated assessments, plans, activities and management.

- Strong senior management endorsement to allow access to information, sites and staff as requested by BNG Team.

- Observe confidentiality where required.

- Guide on projectspecific terminology, systems and teams.

\section{IUCN}

\section{REPRESENTATIVE}

- Staff member of IUCN Member organization or IUCN Commission member of relevant country or region.

- Encourage access \& information sharing with IUCN network.

- Alignment with IUCN Policies and Resolutions.

- Provide additional technical capacity on BNG-related issues where required. 


\section{Stages for a BNG Review Process}

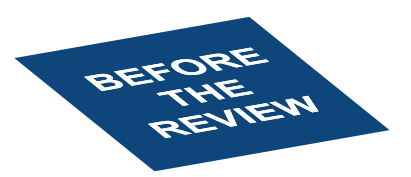

\section{BEFORE THE REVIEW - agreements \& terms of reference}

- Formal agreement on need for BNG Review.

- Engage project staff to provide notice of review facilitate buy-in by key project representatives, and agree on formation and disclosure approach for reporting on review.

- Define terms of reference for review and team members.

- Assemble BNG Review Team and project representatives.

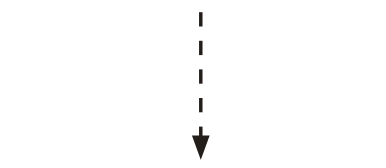

PRE-VISIT - desktop review \& identification of sites/stakeholders to be visited

- Adequacy of data sources.

- BNG assessments, plans and forecasts.

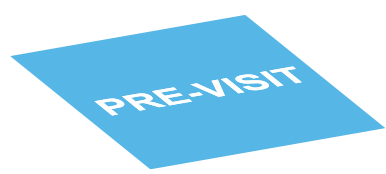

- BNG-related assumptions.

- BNG-related targets and actions in place.

- Reported progress resulting from actions in place.

- Adequacy of team access to BNG assessments/monitoring plans and reports.

- Adequacy of BNG plans, assessments/monitoring plans and forecasts.

\section{VISIT - on-site review \& confirmation}

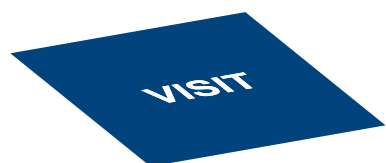

- Integration of BNG-related plans into overall site strategies and plans.

- Stakeholders and partners for BNG.

- On-site appraisal of reported BNG actions.

- Sufficiency of monitoring to assess BNG outcomes.

- On-site appraisal of progress to BNG for each priority biodiversity feature.

\section{POST-VISIT - feedlback \& preparation for future reviews}

- Compile report on findings and recommendations.

- Allow review by team members and project staff.

- Provide final feedback to project and relevant stakeholders.

- Compile supporting information and store report in order to facilitate future reviews.

- Observe confidentiality where required. 


\section{The BNG Review Protocol}

\subsection{What is BNG?}

The concept of minimizing impacts on environment and society has been long established in some sectors (e.g. mining and other extractive industries). In recent years, however, there has been a growing expectation from civil society, regulators and investors for developers, both in private and public sector, to not only do no harm but, in some cases, to also contribute to local, national or global targets for environmental and social wellbeing.

Within the context of biodiversity, this has led to the establishment of measurable targets for mitigation of development impacts that would result in a net maintenance of biodiversity features, resulting in "no net loss", or a net improvement of biodiversity features known as "net gain" or "net positive impact". This simple definition is however subject to a number of established principles and good practice for when and how such targets can be successfully delivered. These include defining a clear reference level based on the status of biodiversity under a pre-project or no project scenario, as well as implementing mitigation actions according to a hierarchy that flows from avoidance to restoration and offsets, as final options. Biodiversity Net Gain (BNG) or Net Positive Impact (NPI) have become popular umbrella terms for these approaches and a number of guidance documents, reviews and policy statements have been produced by IUCN and partners that explore this concept and its appropriate application.

\subsection{What is the BNG Review Protocol?}

The BNG Review Protocol grew out of a stated need by developers and civil society for having greater assurance around the risks and likelihood of implementing the mitigation hierarchy to achieve a net gain for biodiversity within a specific development project (e.g. a mine, a plantation, or a forestry concession). Given the time required to deliver BNG, there was a need to look not only at BNG outcomes, but also the planning and progress towards these outcomes and the status of supporting systems and resourcing required. This required an ability to consistently review BNG implementation at various stages through a project's lifespan, whether at early design or advanced implementation stages.

In order to provide external assurance, the Protocol is explicitly designed to be implemented by independent external experts but may also be adapted for internal monitoring needs that may take place in between more formal, external reviews.

The Protocol is thus intended as a tool for projects and project assessors to use when developing and undertaking a review process to assess BNG implementation. At a minimum, it should be made available to project reviewers. It provides a template to be followed for BNG Reviews involving past and current actions, as well as future plans, associated with the achievement of BNG at the project.

\section{Box 1: The IUCN-Rio Tinto review process}

The BNG Review Protocol draws from the valuable work of the IUCN-Rio Tinto NPI Panel, which ran from 2011-2014. The primary objective of the panel was to develop and test, through a series of pilot reviews of active Rio Tinto sites, a method for independent review of progress towards biodiversity net gain. The panel was supported by selected experts from IUCN and Rio Tinto that were familiar with this approach. The pilot tests included active mining projects in Australia, Madagascar and Mongolia. 
While the Protocol is generic, and designed to promote a standard approach for all BNG Reviews, some elements may not be relevant to all projects. The Protocol requires reviewers to be familiar with the specific policies, strategies and plans relevant to BNG at a project, as well as biodiversity conservation issues in general.

Projects may frame their biodiversity targets and approaches in various ways that may not be easily comparable (e.g. no net loss vs BNG, biodiversity management plans vs environmental management plans). This can be a result of company policies, government regulation or established sector-specific norms. In such cases, reviewers should spend time with the project to ensure they understand the specific terminology and approaches used, which will facilitate the application of the Protocol.

The objective of a BNG Review is an independent evaluation of planning, assessment and review of progress towards, and identification of potential limits, to achieving BNG at a particular project. In some cases, a more detailed investigation of specific issues and progress may be needed as part of the BNG Review and this should be noted within the reviewers' recommendations.

In order to usefully conduct a BNG Review, some minimum information needs to have been produced by the project:

- An assessment of project risks and impacts on biodiversity, including significant residual impacts. This may be in the form of one or more impact assessments, scoping studies, baseline studies or other specialist studies. It is important that such an assessment provide information on biodiversity, particularly priority biodiversity features, and the nature of project impacts on these, including the potential for and scale of any significant residual impacts.

- A forecast of the process and timing of BNG achievement, including monitoring methodology and schedule. Such BNG forecasts are not yet common practice in many sectors, but it is important to have some form of credible technical rationale to justify if, when and how BNG will be achieved as part of the basis for developing management plans. This may range from a credible qualitative assessment based on expert opinion to a quantitative, modelled projection of biodiversity metrics. In either case, such forecasts should be clearly informed by credible biodiversity assessments, and subsequently influence relevant planning by the project. This includes:

$>$ Expected or documented biodiversity losses due to project related impacts.

$>$ Expected timing and extent of biodiversity gains required to achieve BNG via the mitigation hierarchy (avoidance, minimization, rehabilitation and biodiversity offsets).

$>$ Clear definition and justification for social, economic, and environmental assumptions and risks related to expected outcomes of future activities. This includes considering important external changes beyond the project itself, such as climate change, local or national political circumstances, and/or change in local communities support.

$>$ Assessment of the proposed timeline for achieving BNG and a clear judgment in relation to experience elsewhere as to whether the target will be achieved. Inevitably, this will be case-specific and somewhat subjective, but it should be based on background information and mapped uncertainties in the BNG Forecast.

$>$ An assessment of confidence in the BNG forecast. It is expected that this will change over time; and, it should be updated as new information and feedback from monitoring of implementation and outcome of mitigation efforts is received.

- A management plan that describes detailed objectives, tasks, timelines, and task owners related to BNG-related activities. This may be in the form of one or more management plans, depending on the sector (e.g. closure plans, restoration plans, environmental management plans or dedicated biodiversity management plans). Biodiversity Action Plans are the most common vehicle 
for capturing BNG-related actions but not yet widespread in some sectors (e.g. agribusiness). The management plans should follow the mitigation hierarchy in order to be aligned with good practice.

- A Biodiversity Offset Plan, in cases where offsets are considered. This should include aims, objectives, key activities and deliverables for biodiversity offsets at a project. The plan typically identifies the required human and financial resources for delivery of the planned offsets, and it identifies the key risks to achieving the proposed offsets. Such plans may be integrated into broader management plans or stand-alone.

\subsection{How should this BNG Review Protocol be used?}

The Protocol is not a one-size fit all approach. The intent is that the Protocol be used as guidance in the development, implementation and reporting of a fit-for-purpose review process that will be expected to last for the life of a project. While it is strongly recommended that BNG reviews maintain consistency between reviews for any one project, different projects and sectors should carefully consider how to adapt the guidance offered here. This may require significant additional time before the first review to scope the specific needs of the project, consult with project staff and stakeholders, and align with familiar or existing review systems that will encourage uptake of findings and recommendations.

It is at this time that consultation with IUCN may be crucial for delivering a credible Protocol that can be used to review the project. In some cases, customizing the Protocol may need to consider existing reporting requirements (e.g. regulatory compliance audits, certification audits) to reduce redundant assessments and/or to build on existing processes.

It will also be important to agree on transparent criteria for selecting and assembling a review team that is appropriate to the project and/or local circumstances. The final Protocol should be made available to all team members as well as project staff or stakeholders wishing to familiarize themselves with the specific BNG review process, outputs and expectations. 


\section{Preparing for a review}

\subsection{Before the review}

As noted in section 2.3, it is important that the project spend time prior to the first application of the Review Protocol to customize and develop a project-specific, fit-for-purpose approach. With an agreed approach in hand, it becomes imperative that approval and involvement by the project be established as early as possible in order to provide adequate notice of the impending BNG Review to facilitate involvement and buy-in by key project representatives, and to reach agreement on the extent and audience for disclosure of the final review report. At this stage, the focus turns to assembling a team, including the BNG Review Team and project representatives, and defining logistics for ensuring a successful review. An important next step is defining the Terms of Reference for the BNG Review. An example of the Terms of Reference is included in Annex 7.1.

\subsection{The Team}

The team implementing the BNG Review will include the BNG Review Team, as well as a dedicated Project Representative. The BNG Review Team may also include one or more IUCN representatives. This team should include the following roles and responsibilities:

- Team Members - BNG Review Team Members should have expertise in natural and/or social science aspects of biodiversity conservation. They will be expected to support the review throughout, including document review, site visits, and preparation of presentations and reports. BNG Review Team Members should disclose and document any potential for a conflict of interest in undertaking a view, and recuse themselves if necessary. Moreover, in aggregate, the BNG Review Team as a whole should meet the following criteria: i) the BNG Review Team should include at least one member with national, regional, or local biodiversity conservation experience appropriate to the site and issues in question; and ii) the BNG Review Team should strive to be balanced by gender and other locally appropriate considerations relevant to the process.

- Team Lead - The BNG Team Lead should ideally have prior experience of implementing the BNG Review Protocol. Specifically, this experience could comprise either (or both) experience of implementing a previous BNG Review at the site in question, or experience of implementing a BNG Review elsewhere. The BNG Review Team Lead should have prior experience in leadership of technical teams and/or evaluation processes. It is extremely important that the BNG Review Team Lead be identified as soon as possible in order to supervise activities and ensure that the Review Team is thoroughly prepared for what can be a challenging endeavor. It is also highly advantageous for the BNG Team Lead to either have or be able to establish a good working relationship with appropriate project staff in order to facilitate access to the documents and personnel that the BNG Review Team may require. Information confidentially/non-disclosure will in most cases need to be followed. See Annex 8 for more details.

- Project Representative - The Project Representative will be responsible for liaising between the BNG Review Team and the project being reviewed, including managing the logistics of the site visit. The Project Representative should have knowledge of BNG-related assessments, plans, activities and their management. In many cases, they will be the primary source of 
information but this should be supported by other project staff, consultants and stakeholders in order to deliver a credible review. The representative should have strong endorsement from senior management to allow timely access to information, sites and staff as requested by the BNG Team. The representative will also be an important guide to the BNG Team in terms of project-specific terminology, systems and teams.

- IUCN Representative - The BNG Review Team should include at least one member drawn from the conservation community (e.g., an IUCN staff member, IUCN Member organization or an IUCN Commission member) with relevant regional or national experience where the site in question is located. This is also important for encouraging access and information sharing within the IUCN network, alignment with IUCN Policies and Resolutions, and providing additional technical capacity on BNG-related issues where required. The IUCN Representative will also be important for supporting appropriate customization of the BNG Review Protocol to the project in question prior to the first review.

The specific selection process to be followed must be discussed and agreed before the first review is implemented. A typical selection process might include the following steps:

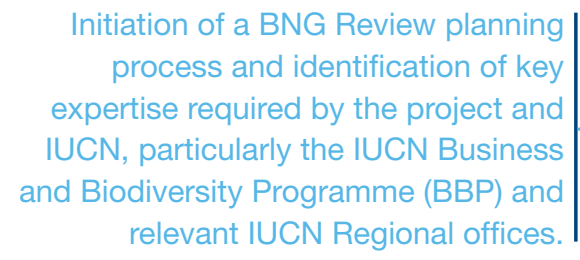

BBP approach and seek commitment from the candidate for the role of BNG Review Team Lead, sharing the Terms of Reference for the BNG Review (Annex 10.1) and Terms of Reference for the BNG Review Team Lead (Annex 10.2) accordingly. If the candidate is not able to serve the role, return to Step 2.

BNG Review Team Lead approaches and seeks commitment from the candidates to serve as a Review Team Member, in order of preference, until at least two additional BNG Review Team Members have been confirmed.

The relevant project management for the site in question to propose a Project Representative.

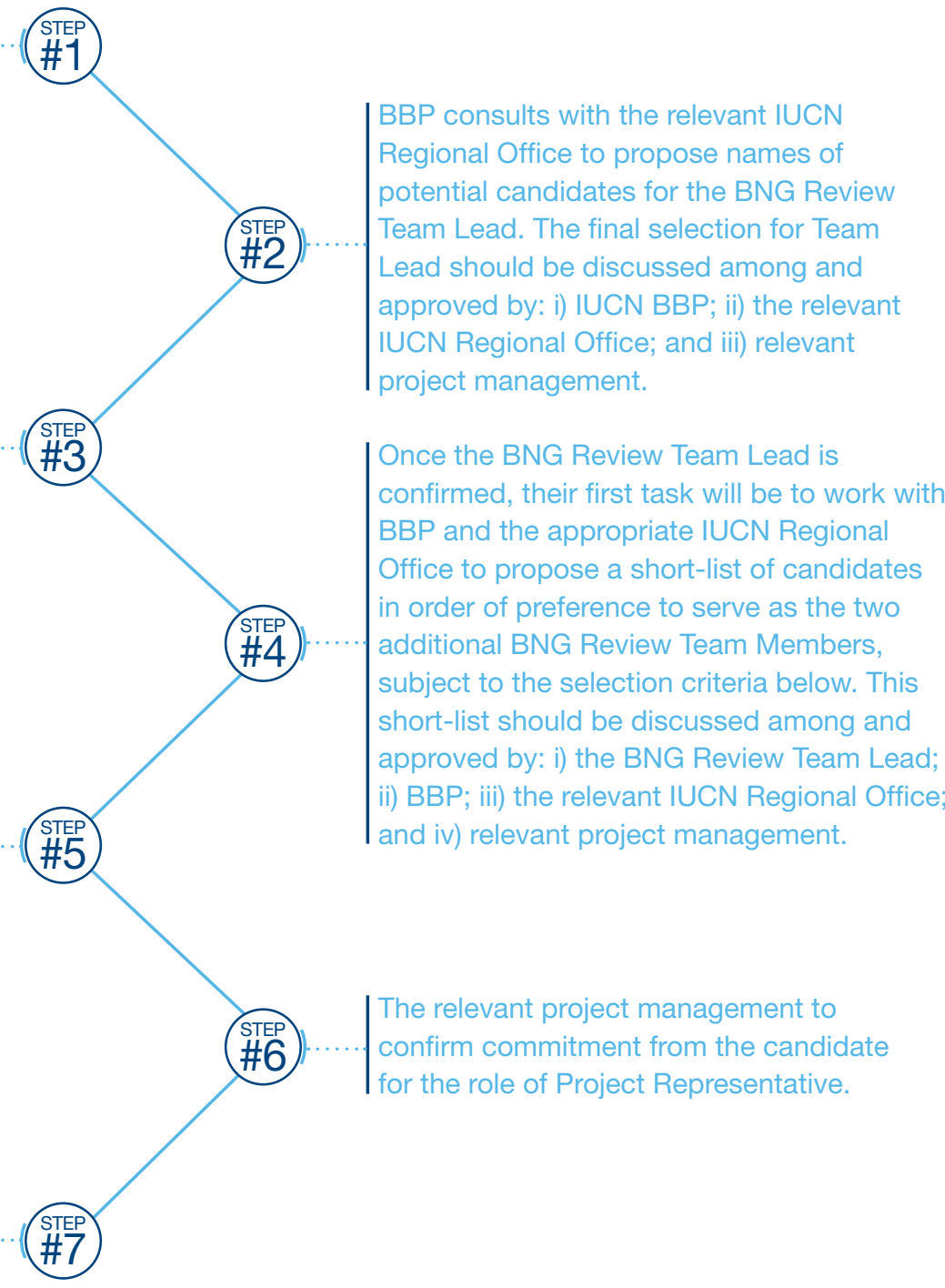

With the team now assembled the BNG Review Process can be initiated. 


\subsection{The Review Process}

The BNG Review process includes the following stages:

- Pre-visit - This stage explores issues to be assessed prior to a site visit via desktop review of existing information. The review focuses on biodiversity-related assessments and plans, previous BNG Review reports (if these have taken place) and reported progress on BNG. This pre-visit review is intended to familiarize the BNG Review Team members with the local context, project management systems and activities, and key strategic issues that may affect progress towards BNG within the agreed timeframe. The pre-site assessment should assist in a preliminary identification of potential issues and further information needs that might require more intensive on-site review or access to specific experts as the BNG Review Team seeks to assess planning for, progress towards, and potential problems with achieving BNG at the site level. It is important to remember that partners and stakeholders requiring interview need to be identified and prioritized so that the key people / organizations can be contacted in advance of a visit.

- Visit - This stage explores issues related to the specific project site(s) being reviewed. It is intended to build an understanding of the factors influencing the project being reviewed, as it works towards delivering BNG. It includes a series of statements intended to act as a prompt to the strategic issues that may be discussed during the site visit. Each area of inquiry/ review commences with an evaluation of the broader management implications associated with the issue and then targets more specific areas of interest. In some instances, the pre-site assessment or initial on-site discussions may point to the need for a more comprehensive review of certain aspects of BNG-related plans, assumptions and actions, including additional interviews with key experts.

- Post-visit - At this stage all necessary information should be compiled and analyzed, including the identification of gaps and recommendations. During this stage any reporting will be delivered, shared and approved between the BNG Review Team and the project representative. Careful documentation of team members, project representatives, external consultations, documents reviewed, and sites visited will be critical to ensuring a credible review which will allow future reviews to be conducted more efficiently, effectively and credibly. This stage also attempts to ensure that openness and transparency, while protecting confidential information; so it documents how and where the review will be shared, thus increasing the opportunity for wider stakeholder buy-in and confidence in the review outputs.

The following sections (Sections 4 and 5) provide examples of the detailed questions to be addressed during various stages of the review. The questions provided should be subject to careful review and agreement before the first review in order to ensure they are appropriate to the project in question. This may relate to the level of risk, nature of BNG implementation, regulatory obligation, stakeholder concerns and expected use for outputs from the BNG Review. Once this is established for the first review, future reviews must follow the agreed approach taken in order to ensure consistency and allow for comparisons of results and monitoring of change over time. However, any subsequent changes reflecting national and financial regulations should be clearly described in a re-visit. 
1.1. Adequacy of data sources
1.1.1. Are the data sources used appropriate, justified, and the best available? Are all cited references available to the BNG Review Team?

1.1.2. Have the BNG-related assessments, plans and forecasts been reviewed internally, and where required, developed in consultation with appropriate, independent regional specialists? If so, is this consultation documented and acknowledged? If not, is there a reason why it was not shared with outside experts for review and comment?

1.1.3. Are there any major uncertainties around the data used that could affect the BNG forecast?
1.2. Adequacy of BNG assessments, plans and forecasts
1.2.1. Has an appropriate project scope been defined and justified, including spatial extent (of infrastructure and of impact)?

1.2.2. Has an appropriate timeframe over which BNG will be achieved, been defined and justified?

1.2.3. What are criteria for identifying priority biodiversity features? If there are any differences between priority biodiversity features used in the BNG-related assessments, plans and/or forecast, are these documented and justified?

1.2.4. Have appropriate metrics for biodiversity been documented and justified? Examples include species populations or a surrogate thereof, extent of habitat, and background rate of loss?

1.2.5. Have baselines for biodiversity metrics been estimated appropriately?

1.2.6. Have the losses and gains been appropriately quantified using these metrics?

1.2.7. Have the losses and gains been quantified following the mitigation hierarchy?

1.2.8. What is the level of confidence associated with the projected trajectory of the metrics?

1.2.9. Are there any major gaps in the BNG-related plans and/or forecast? If so, what are they?

1.2.10. Do BNG-related assessments, plans or forecast account for potential long-term changes in context that could affect BNG-related plans or forecasts (e.g. demographics, climate change, other developments in the area) and the cumulative affects thereof?

\subsection{Adequacy of BNG-related assumptions}

1.3.1. Are the assumptions reasonable and adequately documented regarding background rates of biodiversity loss?

1.3.2. Are the assumptions reasonable and adequately documented regarding additionality?

1.3.3. Are the assumptions reasonable and adequately documented regarding the equivalence of gains and losses, including implementation of mitigation and offsetting measures?

1.3.4. Are the assumptions reasonable and adequately documented regarding the risk of mitigation failure?

1.3.5. Are the assumptions reasonable and adequately documented regarding fungability?

1.3.6. Have the main external and internal stakeholders relevant to BNG-related activities been defined and documented? Who was consulted and how? Stakeholders may include other internal teams, senior project management, biodiversity-related groups and experts (e.g. NGOs, government agencies, and academia) as well as directly affected local communities.

1.3.7. Are assumptions reasonable and adequately documented regarding stakeholder support and critical dependencies for the project, especially government awareness and support for off-site activities? 
1.4. BNG-related targets 1.4.1. Have time-bound and measurable targets been established for each action and actions in place for each priority biodiversity feature?

1.4.2. Do these targets appear sufficient to achieve BNG?

1.4.3. Have clear actions been planned to achieve BNG for each priority biodiversity feature? Do these actions have clear indicators of success, deadlines for delivery and defined owners?

1.4.4. Are these actions, as documented, sufficient to reach the set targets?

1.4.5. What is the documented status of the actions required by BNG-related plans? Planned? Started? On track? At risk? Complete?

1.4.6. Are there any documented potential problems/issues/setbacks with planned or on-going BNG actions?

1.4.7. Are outputs documented to date as expected from the actions designed, or completed within appropriate timeframes to achieve desired progress?

1.5. Reported progress resulting from actions in place
1.5.1. Are adequate and credible data available to justify the documented progress?

1.5.2. Does the documented progress reflect documented milestones in the BNGrelated plans and forecast for each priority biodiversity feature? If not, why not and what issues were encountered?

1.5.3. Are the reductions in threat in line with predictions? If not, why not, and what issues were encountered? What remedial actions have been taken? 


\section{Visit}

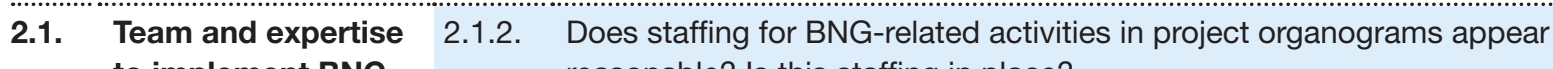
to implement BNG reasonable? Is this staffing in place?

2.1.2. Are staff and consultant qualifications and expertise appropriate?

2.1.3. Are resources adequate to implement BNG-related plans? In cases where offsets are being used, is a long-term financing mechanism in place to support delivery?

2.1.4. Are there plans in place to maintain/build long-term staffing and resourcing?

2.2. Integration of BNG-related plans into overall site strategies and plans

2.2.1. Are internal commitments and obligations for implementing the plans aiming to achieve BNG documented?

2.2.2. Are actions necessary to achieve BNG integrated into necessary project plans and management systems? Is this integration adequate to ensure effective implementation of actions necessary to achieve BNG?

2.2.3. How are BNG-related plans integrated into other relevant project strategies and plans that fall outside the core team responsible for BNG-related actions (e.g. communities or social management systems)? Is this integration adequate to ensure effective implementation of actions necessary to achieve BNG?

\subsection{Stakeholders and} partners for BNG

2.3.1. Have the main external and internal stakeholders relevant to BNG activities been engaged? Is this documented (e.g. agreements, meeting records, other evidence etc.)? If not, why not?

2.3.2. Are terms of reference or other approaches to each partnership adequate to successfully implement actions necessary to achieve BNG?

2.3.3. If a key external or internal stakeholder has not been engaged, what are the risks to achieving BNG?

\subsection{On-site appraisal of reported BNG actions} site appraisal identify any actions or issues that have not been considered in
2.4.1. Does on-site appraisal support the findings of this review of BNG-related assessments, plans, forecasts and related documentation to date? Does onavailable documentation?

2.4.2. Does on-site appraisal confirm that the available documentation on implementation of BNG-related actions to date?

2.4.3. Does on-site appraisal concur that the appropriate internal and external risks to achieving BNG have been identified and that appropriate actions are underway to minimize them? 


\subsection{Analysis of the adequacy of monitoring plans and their execution to assess BNG outcomes}

2.5.1. Has the appropriate internal or external expertise been engaged in support of monitoring?

2.5.2. Are the methods documented and appropriate?

2.5.3. Have appropriate variables been employed in the monitoring program (state indicators, pressure indicators, etc.) that allow tracking of actions and outcomes, particularly with respect to status of priority biodiversity features?

2.5.4. Is monitoring occurring at the appropriate frequency to detect change? Has the monitoring program provided adequate and appropriate coverage to inform BNG progress?

2.5.5. Is there appropriate documentation and reporting on the monitoring program and results?

2.5.6. Do formal feedback mechanisms and schedules exist for monitoring results to be factored into adaptive management of the actions necessary to achieve BNG? Have any updates taken place or been planned for BNG plans or forecasts?

2.6. On-site appraisal of progress to BNG for each priority biodiversity feature
2.6.1. Does the projected trajectory of metrics apparent on-site reach the required target set for the nearest future milestone within BNG-related plans or forecasts?

2.6.2. Is the level of confidence associated with the projected trajectory of the metrics appropriate given considerations on-site?

2.6.3. Are mitigation actions (avoidance, minimization, rehabilitation/restoration and preparation for this, and offsets) apparent as being implemented as scheduled or, if not, why not? 


\section{Post-visit}

On completion of the visit, the BNG Team will be expected to produce a final report. The final format for this report should be discussed and agreed with between IUCN and the project prior to commencement of the BNG Review. Final report format should be clearly aimed at documenting key findings and recommendations in the most actionable way for the project and so ensure uptake of results. This may range from a simple presentation to a written report of findings and recommendations to a fully completed table based on the detailed issues and indicators. It is strongly recommended that findings and recommendations are captured with sufficient detail to ensure that future action or lack thereof can be assessed. This should include establishing roles and responsibilities, timelines and indicators of completion wherever possible.

Regardless of the final format, the following supporting information or meta-data should be captured. This supporting information is important to supporting the credibility of the review and enabling future reviews to build from previous reviews.

Example of minimum supporting information to be recorded for each BNG Review.

\begin{tabular}{|c|l|l|l|l|l|}
\hline \multicolumn{2}{|l|}{ BNG REVIEW PROTOCOL: TEAM DETAILS } & \multicolumn{3}{l}{ [BNG REVIEW DATE 1] } \\
\hline ISsue & Indicator & $\begin{array}{l}\text { Roles \& } \\
\text { Responsibilites }\end{array}$ & Full name & Affiliation & Email \\
\hline
\end{tabular}

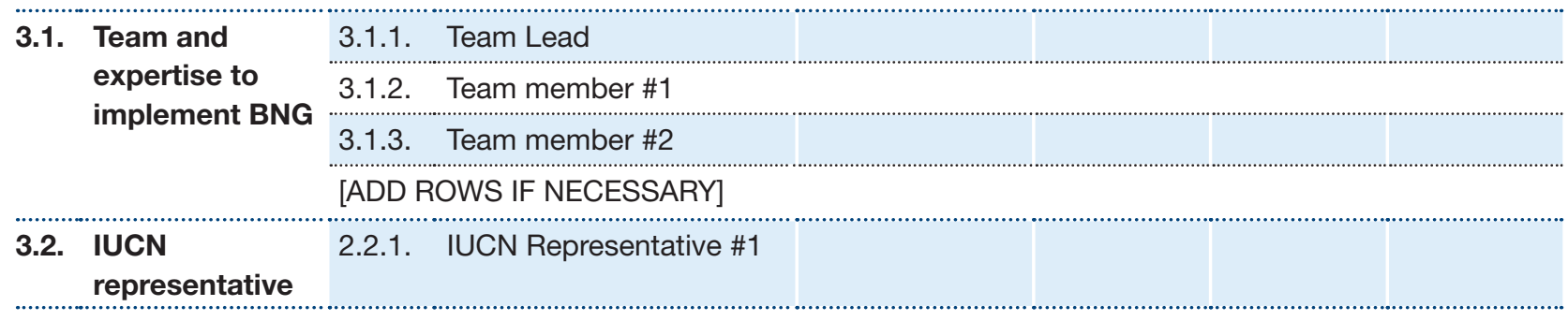

3.3. Project 2.3.1. Project Representative \#1

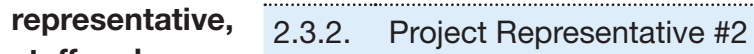

staff and 2.3 .3 . Project Representative \#3

consultants [ADD ROWS IF NECESSARY]

3.4. Additional

3.4.1. Interviewee \#1

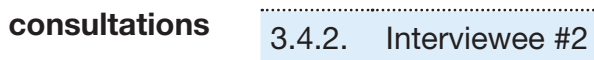

3.4.3. Interviewee \#3

3.4.4. Interviewee \#4

3.4.5. Interviewee \#5

3.4.6. Interviewee \#6

3.4.7. Interviewee \#7

[ADD ROWS IF NECESSARY] 


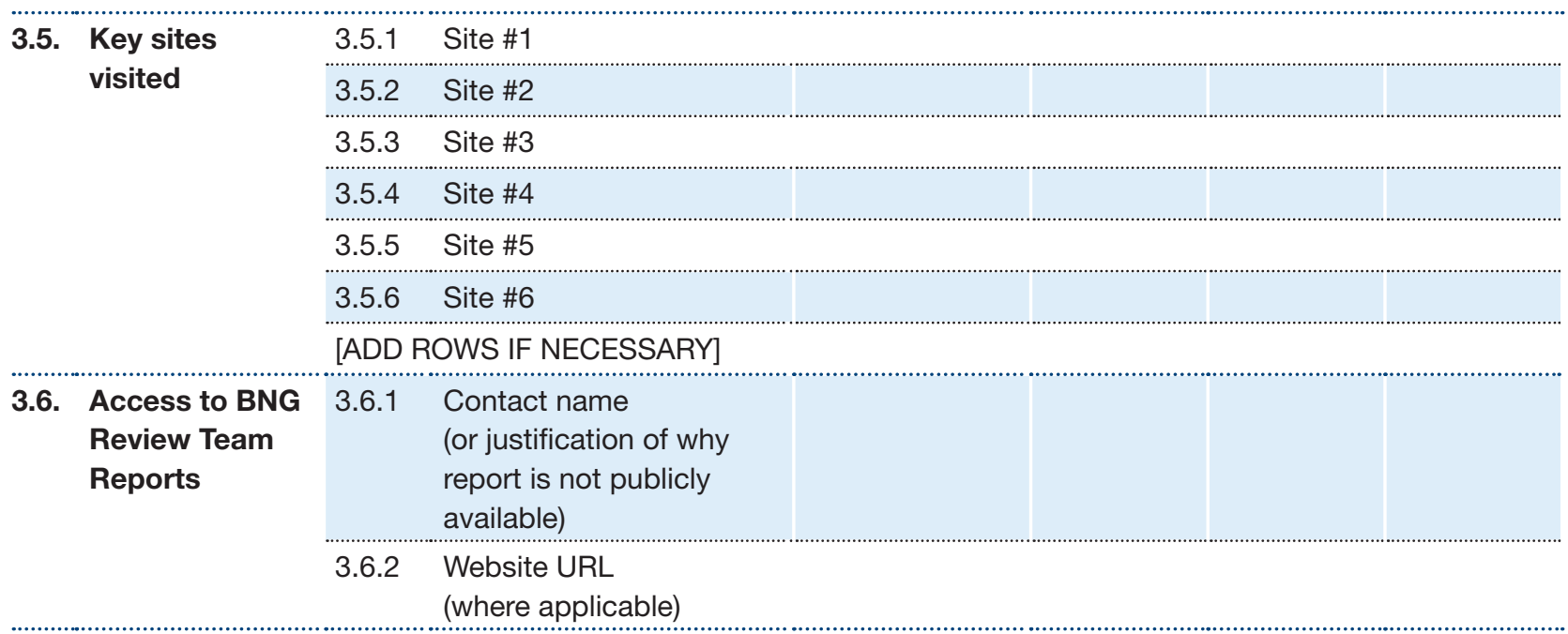

Sufficient time should be allowed for review by the BNG Review Team, the project and IUCN in order to arrive at a final approved report. This should be coordinated by the Team Lead. If issues regarding approval of the BNG Review report exist, these should be documented to the mutual agreement of the BNG Team Members and BNG Review Team Lead. The Team should work on the basis of consensus, but where this is not possible the Team Lead will serve as the final decision maker. Remaining Team Members, IUCN and/or the Project Representative are also welcome to document any significant differences of opinion as an addendum to the final report.

Finally, it is important that BNG Reviews, or at least summaries thereof, are made available to external stakeholders in the interests of transparency. The details of how much, when and the final vehicle for such sharing should be discussed and agreed during the early planning of the review, ensuring that all confidentiality requirements are met. 


\section{Glossary}

Relevant literature may contain different definitions of the terms used throughout this document. The aim of this glossary is not to determine a unique definition for the terms set out here, but rather to enable the reader to understand how terms are used in this Protocol. Further detail and full citations can be found in the IUCN study, Biodiversity Offsets Technical Study Paper.

Additionality

Assurance
The need for a compensation measure to provide a new contribution to conservation, additional to any existing values, i.e. the conservation outcomes it delivers would not have occurred without it.

A positive declaration intended to give confidence; to promise or pledge; guaranty; surety; in this case, assurance of the progress made towards Biodiversity Net Gain.

Baseline A description of existing conditions to provide a starting point (e.g. pre-project or no-project condition of biodiversity) against which comparisons can be made (e.g. post-impact condition of biodiversity), allowing the change to be quantified.

Background rate of loss A description of expected conditions in the project zone in the absence of project activities. It can be framed around species' population, extent of habitat or (preferably) both. As the BNG forecast will be measured against this 'without-project' reference scenario to prove the BNG status, the project proponents must develop a defensible and well-documented 'without-project' reference scenario that must: (1) describe the most likely land-use scenarios without the project activities, and justify why the land-use scenario selected is most likely, (2) describe how the 'without project' reference scenario would affect biodiversity in the project area. This may require input and/or agreement from external experts and other relevant stakeholders.

Biodiversity Net Gain (or Net Positive Impact)
A target for a development project in which the impacts on biodiversity caused by the project are balanced or outweighed by measures taken to avoid and minimise the project's impacts, to undertake on-site restoration, and finally, to offset the residual impacts, so that no loss remains. Where the gain exceeds the loss, the term 'net gain' (NG) may be used instead of no net loss. 


\section{Biodiversity Net Gain Forecast}

A credible rationale, based on qualitative expert opinion and/or quantified modelling of metrics, to justify if, when and how BNG will be achieved at a project site. This should include:

- Expected or documented biodiversity losses due to project related impacts.

- Expected timing and extent of biodiversity gains required to achieve BNG via the mitigation hierarchy (avoidance, minimization, rehabilitation and biodiversity offsets).

- Clear definition and justification for social, economic, and environmental assumptions and risks related to expected outcomes of future activities. This must consider important external changes beyond the project itself, such as climate change, change in local or national political circumstances, and/or change in local communities support.

- Assessment of the proposed timeline for achieving BNG and a clear judgment in relation to experience elsewhere as to whether the target will be achieved. This, inevitably, will be case-specific and somewhat subjective, but should be based on background information and mapped uncertainties in the BNG Forecast.

Confidence in the BNG forecast will change over time and the assessment needs to be included as the forecast is revised due to new information and feedback from monitoring of implementation and outcome of mitigation efforts.

Biodiversity offsets

Biodiversity offsets are measurable conservation outcomes resulting from actions designed to compensate for significant residual adverse biodiversity impacts arising from project development after appropriate prevention and mitigation measures have been taken. The goal of biodiversity offsets is to achieve NNL and preferably a NG of biodiversity on the ground with respect to species composition, habitat structure, ecosystem function and people's use and cultural values associated with biodiversity.

\section{Compensation}

Measures to recompense, make good or pay damages for loss of biodiversity caused by a project. In some languages 'compensation' is synonymous with 'offset', but in this paper 'compensation' is a more general term of which biodiversity offsets are just one subset. Compensation may achieve NNL/NG (in which case it is an offset), but in other cases, compensation can involve reparation that falls short of achieving no net loss (and is therefore not an offset). This can be for a variety of reasons, including that the conservation actions were not planned to achieve no net loss; that the residual losses of biodiversity caused by the project and gains achievable by compensation are not quantified; that no mechanism for long term implementation has been established; that it is impossible to offset the impacts (for instance, because they are too severe or pre-impact data are lacking, so it is impossible to know what was lost as a result of the project); or that the compensation is through payment for training, capacity building, research or other outcomes that will not necessarily result in measurable conservation outcomes on the ground. 
Ecological equivalence In the context of biodiversity offsets, this term is synonymous with the concept of 'like for like' and refers to areas with highly comparable biodiversity components. This similarity can be observed in terms of species diversity, functional diversity and composition, ecological integrity or condition, landscape context (e.g., connectivity, landscape position, adjacent land uses or condition, patch size, etc.), and ecosystem services (including people's use and cultural values).

Exchange rules $\quad$ A set of rules established by policy makers or offset planners to define which components of biodiversity can and cannot be substituted for others in a biodiversity offset, and how such substitutions can occur. These rules may be explicit, or they may be implicit within the definitions adopted of biodiversity offsets and associated requirements, such as 'like for like' and 'trading up'.

Fungibility

The ability to substitute or exchange one item with another identical item. An item is fungible if it is directly convertible for or replaceable by another of like nature or kind. Fungibility, as a concept in conservation science, and specifically relating to biodiversity offsets, refers to the validity of exchanging one biodiversity value with another like-value in a different space or time. This concept is commonly referred to as 'like-for-like'. See also 'Ecological equivalence'.

Like-for-like or better See 'Ecological equivalence', 'Fungibility' and 'Trading up'

Metrics

A set of measurements that quantifies results. In this Protocol, we consider metrics (or currencies) to be the unitary measures of biodiversity lost, gained or exchanged. These vary from very basic measures such as area, to sophisticated quantitative indices of multiple biodiversity components which may be variously weighted.

Mitigation hierarchy The mitigation hierarchy comprises:

- Avoidance: measures taken to avoid creating impacts from the outset, such as careful spatial or temporal placement of elements of infrastructure, in order to completely avoid impacts on certain components of biodiversity.

- Minimisation: measures taken to reduce the duration, intensity and / or extent of impacts that cannot be completely avoided, as far as is practically feasible.

- Rehabilitation / restoration: measures taken to rehabilitate degraded ecosystems or restore cleared ecosystems following exposure to impacts that cannot be completely avoided and / or minimised.

- Compensation or Offset: measures taken to compensate for any residual significant, adverse impacts that cannot be avoided, minimised and / or rehabilitated or restored. Measures to achieve NNL or a NG of biodiversity for at least as long as the project's impacts are biodiversity offsets. Offsets can take the form of positive management interventions such as restoration of degraded habitat, arrested degradation or averted risk, where there is imminent or projected loss of biodiversity. Measures that address residual impacts but are not quantified to achieve NNL or not secured for the long term are compensation, otherwise known as compensatory mitigation. 


\begin{tabular}{|c|c|}
\hline Mitigation measures & full set of activities covering the entire mitigation hierarchy. \\
\hline $\begin{array}{l}\text { No Net Loss and a } \\
\text { Net Gain }\end{array}$ & $\begin{array}{l}\text { A target for a development project in which the impacts on biodiversity caused } \\
\text { by the project are balanced or outweighed by measures taken to avoid and } \\
\text { minimise the project's impacts, to undertake on-site restoration and finally to } \\
\text { offset the residual impacts, so that no loss remains. Where the gain exceeds } \\
\text { the loss, the term 'net gain' (NG) may be used instead of no net loss. }\end{array}$ \\
\hline $\begin{array}{l}\text { Non-offsetable } \\
\text { threshold and Non- } \\
\text { offsetable } \\
\text { impacts }\end{array}$ & $\begin{array}{l}\text { This is a level of severity beyond which impacts on biodiversity by a } \\
\text { development project may no longer be capable of being offset. For example, } \\
\text { it is not possible to offset the global extinction of a species. Levels of } \\
\text { irreplaceability and vulnerability of the biodiversity components to be affected } \\
\text { by the project, and the degree of uncertainty with respect to severity of } \\
\text { impacts and the probability of success of a biodiversity offset, are all likely } \\
\text { to be material factors in determining whether impacts on biodiversity can } \\
\text { be offset. }\end{array}$ \\
\hline Offset & See Biodiversity offset \\
\hline $\begin{array}{l}\text { Priority biodiversity } \\
\text { features }\end{array}$ & $\begin{array}{l}\text { Features occurring within and outside a project's direct area of control or } \\
\text { influence, including species of conservation value, sensitive habitats, protected } \\
\text { areas and other stakeholder identified biodiversity which are considered to be } \\
\text { a priority for management. Various approaches to defining biodiversity features } \\
\text { exist and will vary depending on corporate policy, investor performance } \\
\text { standards or safeguards, stakeholder concerns and/or national regulation. }\end{array}$ \\
\hline
\end{tabular}

Trading up (or 'like-for-like or better')
Conserving through an offset components of biodiversity that are a higher conservation priority (e.g. because they are more irreplaceable and vulnerable) than those affected by the development project for which the offset is envisaged. 


\section{Annexes}

\subsection{Terms of Reference for a BNG Review Team}

Objective The BNG Review will review BNG planning, implementation and outcomes at [PROJECT SITE, COUNTRY]. The sites included in the BNG Review include [NAMES, LOCATION]. The following aspects will be excluded from this review: [ISSUES OR SITES]. This will be the [RANK E.G. FIRST? SECOND?] BNG Review conducted. The last BNG Review conducted was on [DATE] and is available via [SOURCE].

Team The BNG Review Team will require the close coordination and collaboration between all members. Key supporters of the BNG Review Team include: [DESCRIBE SUPPORTERS AFFILIATIONS WITHIN IUCN AND PROJECT]. These key supporters will select the BNG Review Team Lead from among qualified leads chosen according to the Selection Criteria and following the Selection Process (Annex). These supporters and the chosen BNG Review Team Lead will select the rest of the BNG Review Team Members, according the recommendations in the BNG Review Protocol. Terms of reference for the BNG Review Team Lead and Members are included in (Annex 0 and 0 ).

The supporters and BNG Review Team designated to deliver this BNG Review will be: [LIST SUPPORTERS NAMES, AFFILITATION].

Term Depending on the size of the project, the BNG Review will take place over an agreed period of time, from acceptance of the TOR on [DAY/MONTH/YEAR]. The intervals between any subsequent visits will also be agreed

Activities \& The BNG Review process will include Deliverables

Pre-Visit $\quad$ Project Representative initiates BNG Review planning process (at least 4 months prior to BNG Review commencement).

- Confirm BNG Review Team \& key supporters, including finalization and acceptance of Terms of Reference for BNG Review (this document), BNG Review Team Lead and Members.

- Review and confirm calendar for key activities.

- Key documents will be shared with BNG Review Team Lead by Project Representative (at least 4 months prior to Visit). These key documents include [EDIT AS NECESSARY].

- Implement all pre-visit activities described in Terms of Reference for BNG Review Team Lead and Members. 
Visit $\quad$ Implement all Visit activities described in Terms of Reference for BNG Review Team Lead and Members.

- Deliver introductory presentation to project site staff, including [NAME KEY STAFF], describing:

- BNG background

- Visit objectives \& itinerary

- BNG Review Team

- Deliver close-out meeting to site staff, including [NAME KEY STAFF], describing:

- Key impressions, findings, observations

- Next steps

- Visit will include [EDIT AS NECESSARY]:

- Day 1: Arrive \& acclimatize

- Day 2: Draft Pre-visit review

- Day 3: Opening meeting, on-site reviews

- Day 4: On-site reviews

- Day 5: Draft Review Report, Close-out meeting

- Day 6: Deliver hard and electronic copies of draft Review Report

- Day 7: Depart

Post-Visit o Implement all post-visit activities described in Terms of Reference for BNG Review Team Lead and Members.

- Project Representative/s to share responses to Draft BNG Review Report.

- BNG Review Team Members to finalize BNG Review Report. This may include discussions with Project Representative/s, as necessary.

o Project Representative to approve final Review Report.

- BNG Review Team Lead to approve Review Report on consultation with BNG Review Team. 


\subsection{Terms of Reference for a BNG Review Team Lead}

Objective The BNG Review Team Lead will represent IUCN as leader of the BNG Review as well act as expert reviewer of BNG planning, implementation and outcomes at [PROJECT SITE, COUNTRY]. Further details on the BNG Review are included in the Terms of Reference for the BNG Review (see Annex 7.1).

Term The BNG Review Team Lead will lead the BNG Review Team and support the BNG Review process for a maximum of [NUMBER E.G. 25] works days, spread over a period of [NUMBER E.G. 10-12] months from acceptance of the TOR.

Activities \& The BNG Review Team Lead will be expected to:

Deliverables

Pre-Visit $\circ$ Review and select BNG Team Members in collaboration with IUCN (10 days) and Project Representative.

- Coordinate with designated Project Representative to:

- Discuss and agree on Terms of Reference for BNG Review.

- Plan and confirm Visit logistics, including travel dates, requirements, accommodation, vaccinations and/or interpreter assistance. Logistics should be confirmed at least 2 weeks prior to Visit.

- Confirm calendar of activities and deliverables for BNG Review process, including pre, during and post-Visit.

- Agree on issues of confidentiality and non-disclosure.

- Initiate acquisition of key documents, at least four months prior to a scheduled visit.

- Acknowledge receipt of key documents and request additional information as necessary.

- Coordinate with BNG Review Team Members to:

- Contact and confirm availability of proposed BNG Review Team Members.

- Disseminate and confirm agreement on Terms of Reference for BNG Team Members.

- Confirm Visit logistics, including travel dates, requirements, accommodation, vaccinations and/or interpreter assistance.

- Confirm availability of BNG Review Team Members regarding virtual and in-person meetings, including travel related to the Visit.

- Coordinate dissemination and confirm receipt of key documents to BNG Review Team Members.

- Identify and prioritize additional information resources (e.g. documents, organizations, experts and/or stakeholders) required for comprehensive BNG Review, as requested by BNG Team Members.

- Coordinate access to additional information resources (e.g. documents, organizations, experts and/or stakeholders) required for comprehensive BNG Review, as requested by BNG Team Members. This includes contact with required individuals and/or organizations to schedule interviews pre or during Visit, as appropriate.

- Provide expert review of key documents. 
Visit $\quad$ Coordinate and participate fully in Visit, including key meetings, (5+ days) presentations and interviews.

- Coordinate development and delivery to Project Representative/s of hard and electronic copies of: Draft BNG Review Report, Introductory presentation, and Close-out presentation.

- Coordinate with Project Representative/s to incorporate responses to Draft BNG Review Report within agreed time frame.

- Coordinate with BNG Review Team Members to finalize and approve BNG Review Report. If issues regarding approval of the BNG Review Report exist, these will be documented to the mutual agreement of the BNG Team Members and BNG Review Team Lead.

- Serve as final decision maker for BNG Review Team in the event of a lack of consensus. The BNG Review Team, however, will work on the basis of consensus as far as is possible.

- Coordinate with Project Representative/s to approve final BNG Review Report.

- Coordinate delivery of appropriate BNG Review documentation to IUCN. 


\subsection{Terms of Reference for a BNG Review Team Member}

Objective The BNG Review Team Member will represent IUCN as an expert reviewer of BNG planning, implementation and outcomes at [PROJECT SITE, COUNTRY]. Further details on the BNG Review are included in the Terms of Reference for the BNG Review Team (see Annex 7).

Term The BNG Review Team Member will support the BNG Review process for a maximum of [NUMBER E.G. 14] works days, spread over a period of [NUMBER E.G. 10-12] months from acceptance of the TOR.

Activities \& The BNG Review Team Member will be expected to:

Deliverables

Pre-Visit (3-5 days)

○ Confirm availability with the BNG Review Team Lead regarding virtual and in-person meetings, including travel related to the Visit.

- Acknowledge receipt of key documents.

- Provide expert review of key documents as agreed with BNG Review Team Lead.

- Identify and prioritize additional information resources (e.g. documents, organizations, experts and/or stakeholders) required for comprehensive BNG Review. These will be communicated to BNG Review Team Lead to allow access before or during Visit.

Visit $\quad \circ$ Participate fully in Visit, including key meetings, presentations and (5+ days) interviews as agreed with BNG Review Team Lead.

- Collaborate with BNG Review Team Lead and other Team Members to develop and present opening and close-out presentations, and draft BNG report.

- Collaborate with BNG Review Team Lead and Team Members to finalize BNG Review Report.

- Approve final BNG Review Report. If issues regarding approval of the BNG Review Report exist, these will be documented to the mutual agreement of the BNG Team Member and BNG Review Team Lead. 



\section{IUCN}

INTERNATIONAL UNION

FOR CONSERVATION OF NATURE

WORLD HEADQUARTERS

Rue Mauverney 28

1196 Gland, Switzerland

mail@iucn.org

Tel +41229990000

Fax +41 229990002

www.iucn.org 